\title{
Reflecting optical system to increase signal intensity in confocal microscopy
}

\author{
DongKyun Kang*, JungWoo Seo, DaeGab Gweon \\ Nano Opto Mechatronics Laboratory, Dept. of Mechanical Engineering, Korea Advanced Institute \\ of Science and Technology, Taejon, Korea
}

\begin{abstract}
In fluorescence mode confocal microscopes, only $0.02 \%$ of emitted signal can be detected in best case. So, we proposed reflecting optical system to increase signal intensity detected in photon detector. In this paper, we evaluate the proposed reflecting optical system using optical transfer function. To evaluate the proposed system, we used the modeling method based on wave optics. We first calculated point spread function of total system, and calculated optical transfer function of total system. When we use the proposed reflecting optical system, we can increase the signal intensity detected in photon detector. Amount of increased signal intensity depends on the ratio of NA of objective in the original confocal microscopy to NA of objective in reflecting optical system. We also simulated axial response of total system. FWHM of axial response increased a little when using reflecting optical system. The amount of increased FWHM also depends on the ratio of NA, mentioned above. Maximum increase in FWHM of axial response is about 5\%.
\end{abstract}

Keywords: Confocal microscopy, Reflecting optical system, Optical transfer function, Signal intensity

\section{INTRODUCTION}

Confocal microscopy is first invented by M. Minsky. Due to the aid of development of various laser and computer, confocal microscopy is widely used in cell biology, physiology, cytogenetics, diagnostic pathology and the material sciences. ${ }^{1}$ The main advantage of confocal microscopy over conventional optical microscopy is the ability of optical sectioning. With pinhole aperture, light from out of focus plane can be rejected, thus only the thin slice in focal plane can be imaged in the thick specimen. Using this optical sectioning ability, true three-dimensional image of specimen can be achieved.

But confocal microscopy has some disadvantages. It takes time to perform a scan particularly for high resolution imaging over a large area or volume of a specimen. ${ }^{2}$ And it has low optical transfer efficiency, which means only small

*godogo@mail.kaist.ac.kr ; phone 8242 869-3265 ; fax 8242 869-5225 ; http://nom.kaist.ac.kr ; Nano Opto Mechatronics Laboratory, KAIST, Yusung-gu, Taejon, Korea 305-701 
part of emitted fluorescence can be detected. To overcome the low quantum efficiency, we proposed the reflecting optical system before ${ }^{3}$.

In the first part of this paper, we describe the proposed reflecting optical system briefly. And in the next section, we analyze the performance of reflecting optical system.

\section{REFLECTING OPTICAL SYSTEM}

In fluorescence mode confocal microscopy, which is a very popular instrument in biology, optical transfer efficiency is very low ${ }^{4}$. Amount of light emitted from fluorophore is limited by photodynamic property of given fluorophore. Fluorescence photobleaching limits the emitted photons and excited-state saturation limits the available imaging intensity. So, we can't get more emitted photons by increasing intensity of illumination light over fluorescence saturation level. In case of limited available photons, it is very important to detect as many photons as possible to enhance good image quality. For confocal microscopy, optical transfer efficiency, ratio of detected fluorescence to emitted fluorescence, is usually much less than $0.3 \%$ even for maximum-size detector aperture.

There were several methods proposed to increase the amount of detected fluorescence or to increase sensitivity of photon detector. Art (1989) demonstrated a simple trans-illuminated confocal design in which emitted fluorescence propagating forward is captured by second objective and collimated to be reflected by plane mirror ${ }^{4}$. Reflected fluorescence is focused by second objective and collimated again by first objective to be detected by photon detector. S.W. Hell et al. proposed 4Pi-confocal microscopy, which has good axial resolution and increased number of detected photons ${ }^{5}$. But these methods are only applicable to the object scanning. If fast scanning speed is necessary, beam scanning type is preferred, and we need different method to increase detected fluorescence intensity.

As one of the solutions to this problem, we proposed reflecting optical system behind the specimen ${ }^{3}$. Proposed reflecting optical system is described in Fig. 1.

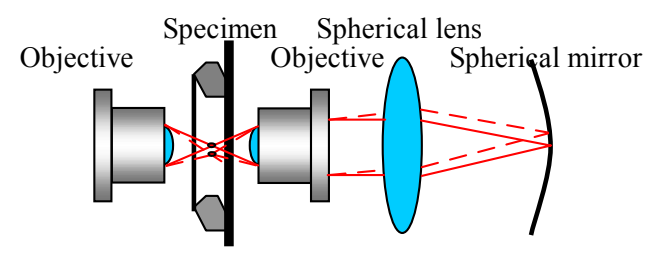

Figure 1 : Schematics diagram of proposed reflecting optical system

Fluorescence propagating forward passes proposed reflecting optical system so that it is focused at the point where it came from. Now, this focused beam passes through the first objective and beam scanning system to reach the pinhole aperture. Because this beam came from where illumination spot was, it can pass through the pinhole and can be detected 
by photon detector. So, detected intensity is increased. This property doesn't depend on specific scanning point, so the proposed reflecting optical system can be used in beam scanning confocal microscopy.

\section{PERFORMANCE OF REFLECTING OPTICAL SYSTEM}

The reflecting optical system proposed in this paper is expected to increase detected intensity. To analyze performance of proposed reflecting optical system, we used the concept of point spread function, optical transfer function, and axial response. In this chapter, we present the modeling of proposed reflecting optical system for wave optical analysis. And we explain the concept of point spread function briefly. At the end of this chapter, we describe the optical transfer function and axial response of proposed reflecting optical system to compare its performance with the confocal microscopy without reflecting optical system.

\subsection{Modeling for wave optical analysis}

Simplified model for beam scanning confocal microscopy with proposed reflecting optical system can be easily understood by Fig. 2. In this model, beam scanning mechanism is replaced with moving light source and moving pinhole aperture which are conjugate to each other. Scanning surface is considered as curved due to the aberration of beam scanning optical system. Reflecting optical system is designed so that the surface of spherical mirror is conjugate surface to the curved scanning surface.

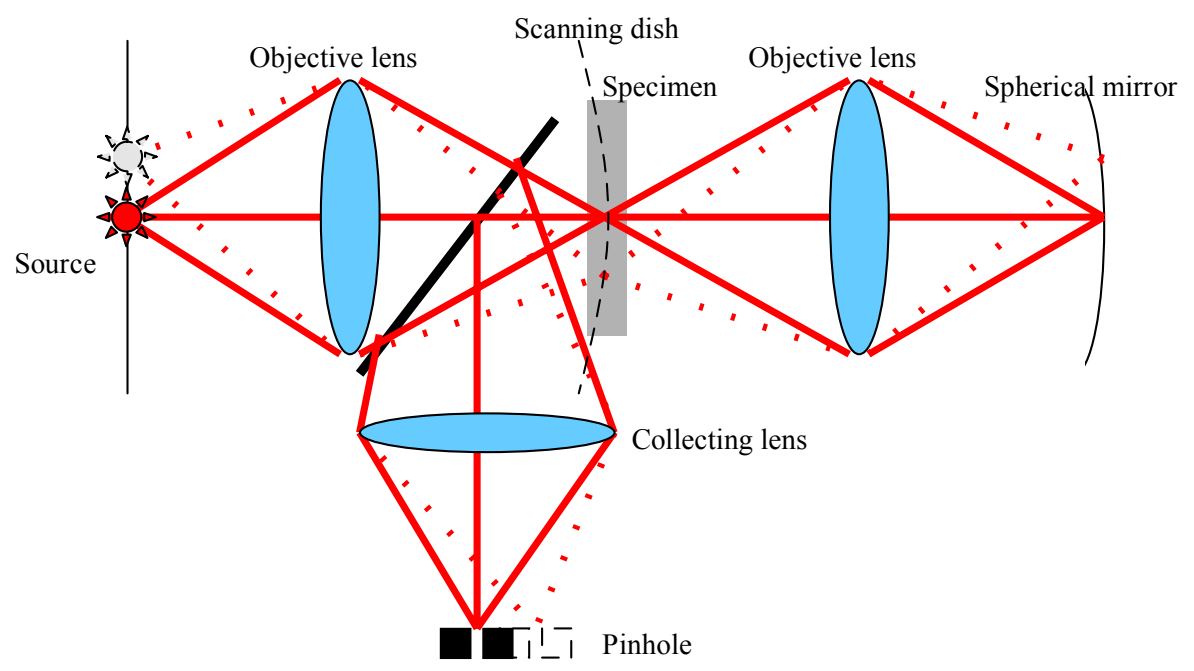

Figure 2 : Simplified model concerning scanning surface 
If we assume that objective has no aberration, the curved scanning surface made by beam scanning optical system can be considered to be made by objective with only field curvature. But if we consider the curved scanning surface as a reference plane, then this field curvature can be eliminated so that scanning surface can be considered simply as a plane $^{6}$. Once scanning surface is considered as a plane, the spherical mirror in reflecting optical system can be considered as a plane, since it is the conjugate plane to the scanning surface. So, simplified model with plane scanning region is described in Fig. 3. We use this simplified model to analyze the performance of reflecting optical system.

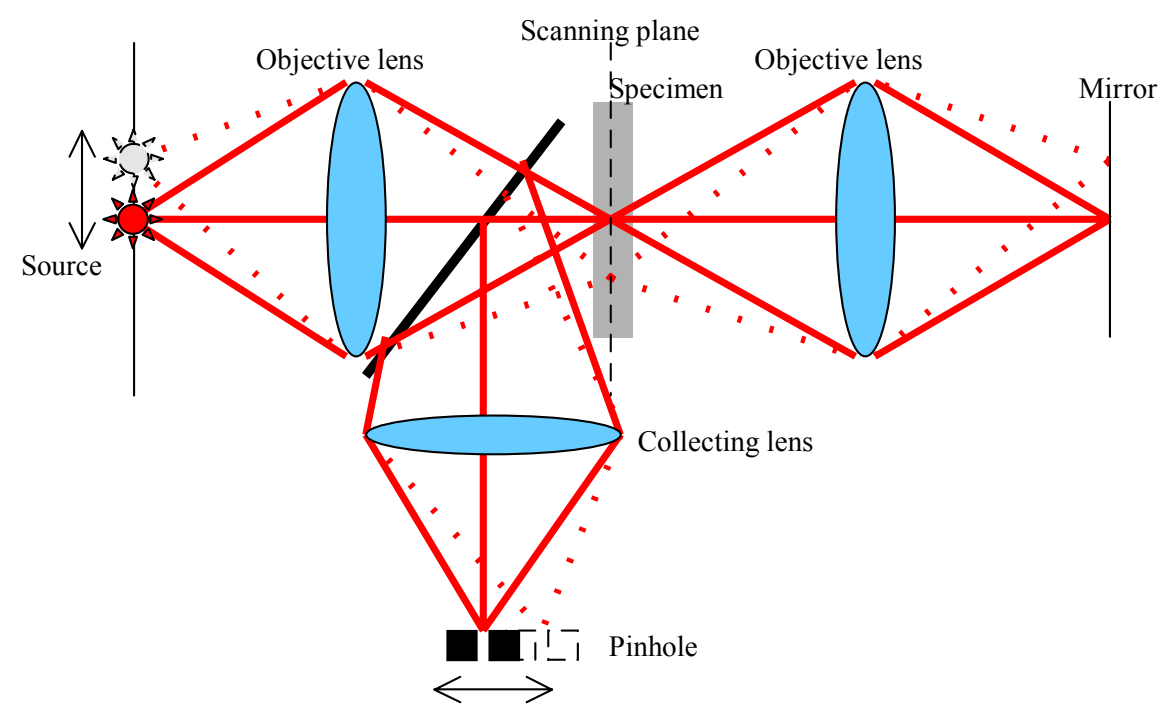

Figure 3 : Simplified model with plane scanning surface

\subsection{PSF of overall optical system with proposed reflecting optical system}

For fluorescence type confocal microscopy, relation between detected intensity and object function for point detector is usually expressed as Eq. $(1)^{7}$.

$$
I\left(\overrightarrow{r_{s}}\right)=\int_{-\infty}^{\infty}\left|h_{1}\left(M \overrightarrow{r_{1}}\right)\right|^{2} o_{f}\left(\overrightarrow{r_{s}}-\overrightarrow{r_{1}}\right)\left|h_{2}\left(\overrightarrow{r_{1}}\right)\right|^{2} d \overrightarrow{r_{1}}
$$

Similar equation can be established for simplified model with reflecting optical system, which we are considering now. 


$$
I\left(\overrightarrow{r_{s}}\right)=\int_{-\infty}^{\infty}\left|h_{i l l u}\left(-\overrightarrow{r_{s}}+\overrightarrow{r_{1}}\right)\right|^{2} o_{f}\left(\overrightarrow{r_{1}}\right)\left(\left|h_{1 \operatorname{det}}\left(-\vec{r}_{s}+\vec{r}_{1}\right)\right|^{2}+\left|h_{2 \operatorname{det}}\left(-\overrightarrow{r_{s}}+\overrightarrow{r_{1}}\right)\right|^{2}\right) d \overrightarrow{r_{1}}
$$

Eq. (2) is different from Eq. (1) in two points. Since we are considering beam scanning type confocal system, coordinate of object function is fixed, while coordinate of illumination and detection point spread function changes which stands for the movement of source and detection pinhole respectively. And detection point spread function is composed of two parts. One is usual detection point spread function for confocal microscopy, and the other is point spread function for detection path with reflecting optical system. If we define $h_{a}$ as overall intensity point spread function, we can explain the equation above as follows.

$$
I\left(\overrightarrow{r_{s}}\right)=o_{f}\left(\overrightarrow{r_{s}}\right) \otimes_{3} h_{a}\left(\overrightarrow{r_{s}}\right)
$$

This shows that there is no difference of imaging process between object scanning and beam scanning type confocal microscopy when we assume the curved surface as reference plane in beam scanning type. Before we consider overall intensity point spread function, detection point spread function with reflecting optical system, $h_{2 d e t}$, must be considered, since this is the main difference to the conventional confocal microscopy.

\subsection{Detection PSF with reflecting optical system}

Coherent transfer function of detection path with reflecting optical system is multiplication of three CTF, which are two CTF of second objective and CTF of collecting lens. CTF is one in central region and zero in outer region. So, CTF with minimum non-zero region will govern the non-zero region of overall CTF. When NA of second objective is larger than that of collecting lens, NA of returning fluorescence from reflecting optical system is limited by that of collecting lens. Otherwise, when NA of second objective is smaller than that of collecting lens, main bottleneck is second objective. If we assume that NA of second objective is not larger than that of collecting lens, CTF of second detection path is just CTF of second objective. (This doesn't lose generality, since second of objective with larger NA will acts as that with the same NA to collecting lens) So, PSF of second detection path is just PSF of second objective. Letting the ratio of second objective NA to collecting lens NA $m$, we can get the equation below for PSF of second detection path.

$$
h_{2 \mathrm{det}}(v, u)=m^{2} h_{1 \mathrm{det}}\left(m v,-m^{2} u\right)
$$


Since $m$ is not larger than one, Eq. (4) means that detection signal by second path, which uses reflecting optical system, can't be larger that that by first path, which is conventional detection path in confocal microscopy. And the shape of PSF is broader than that of first detection path. Using this result we can calculate the optical transfer function and axial response of proposed optical system.

\subsection{Performance of confocal microscopy with reflecting optical system}

Optical transfer function is Fourier transform of intensity point spread function. OTF explains spatial frequency response of given optical system. Practically, it can be calculated by convolution of OTF of illumination path and detection path. Before we actually calculate the OTF, we consider OTF of second path at this point. From the Eq. (4), OTF of second path has the given relation below.

$$
C_{2 \mathrm{det}}(l, s)=C_{1 \mathrm{det}}\left(\frac{l}{m}, \frac{s}{m^{2}}\right)
$$

Eq. (5) shows that OTF of second path has narrower band pass in spatial frequency space, which means it worsen the frequency response property of overall system.

Numerical results of OTF are shown in Fig. 4. Absolute size of OTF is doubled when reflecting optical system is used, but there is no apparent difference of shape and pass band. When $m$ is smaller than one, amount of increase in absolute size is decreased compared to the case when $m$ is one.

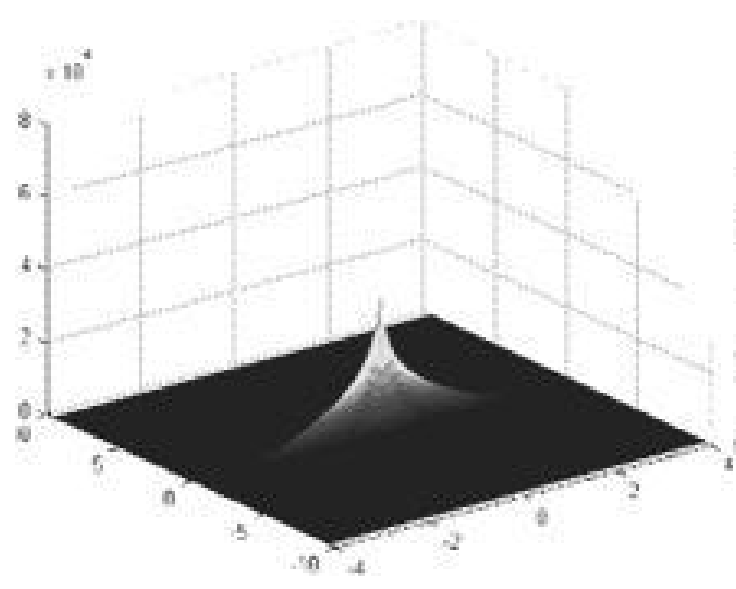

(a) : OTF without reflecting optical system

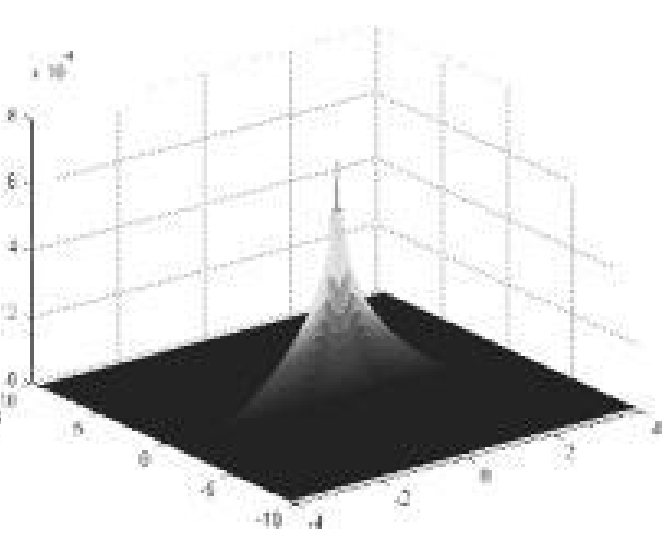

(b) : OTF with reflecting optical system

Figure 4 : OTF of overall optical system (a) : OTF without reflecting optical system (b) : OTF with reflecting optical system 


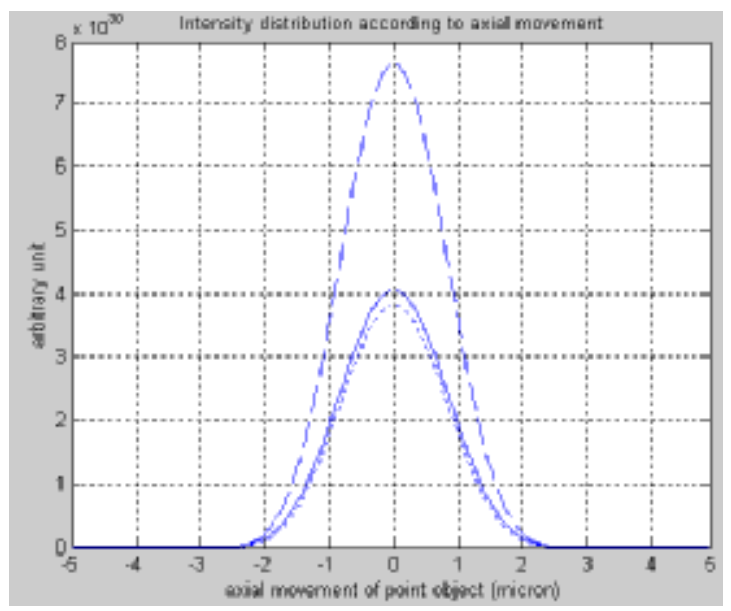

(a) without normalization

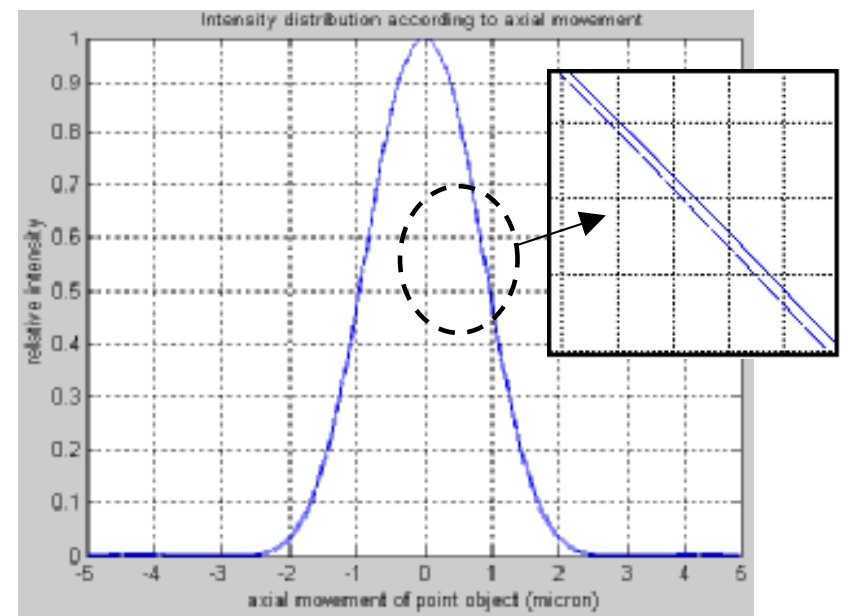

(b) with normalization

Figure 5 : Axial response of overall optical system. (dashed line : $m=1$, solid line $: m=0.5$, dotted line $: m=0$ ) (a) : axial response without normalization, (b) : normalized axial response

Fig. 5 shows axial response of overall optical system. With reflecting optical system, detected intensity increases. But the amount of increase is proportional to $m^{4}$, so when $m=0.5$, there is only $6.25 \%$ of increase. With normalization, there looks no difference according to the change of $m$ value. But magnified view of central lobe shows axial response curve is broader when $m$ is 0.5 . This means FWHM of axial response is increased.

Fig. 6 shows the change of relative FWHM according to the change of $m$ value. As $m$ gets larger, FWHM also gets larger until $m$ is about 0.8 . As $m$ gets larger than 0.8, FWHM gets smaller. In any case, FWHM is increased, which worsen the axial response of overall optical system. The maximum increase of FWHM is about 5\%.

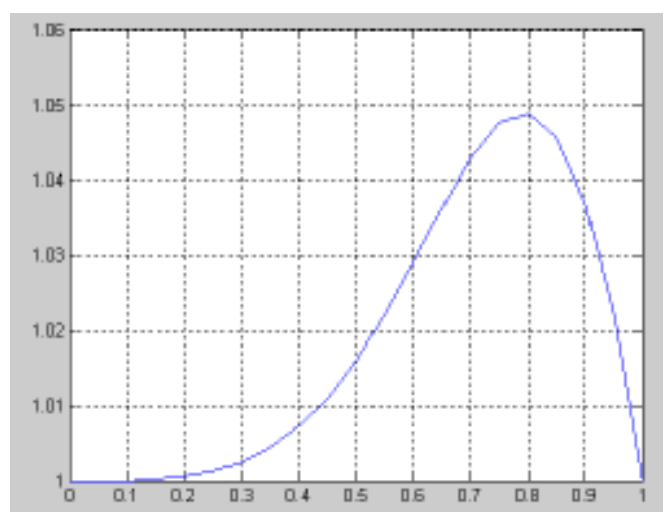

Figure 6 : Relation between relative FWHM with respect to change of $m$ 


\section{RESULT}

We proposed reflecting optical system and analyzed performance of optical system using the concept of PSF, OTF, and axial response. Detected intensity is increased when reflecting optical system is used, and the amount of increase if proportional to $m^{4}$. FWHM of axial response is increased and maximum value of increase is $5 \%$. In actual setup, there is movement of optical probe due to the refractive index change in specimen. To compensate this effect, motorized stage to move second objective in reflecting optical system is necessary.

\section{REFERENCE}

1. C.J.R. Sheppard, D.M. Shotton, Confocal Laser Scanning Microscopy, Springer Verlag, New York, 1997

2. John G. Walker, "Non-scanning confocal fluorescence microscopy using speckle illumination”, Optics Communications. 189, pp. 221-226, 2001

3. D.K. Kang, J.W. Seo, Dae-gab Gweon, "Signal increasing method in confocal scanning microscopy in fluorescent mode using curved mirror", Proceeding of International conference on control, Automation and Systems 2001, 1572-1575, Oct. 2001

4. J.B.Pawley (Ed.), Handbook of Biological Confocal Microscopy, Plenum Publishers, New York, 1995

5. K. Bahlmann, S. Jakobs, S.W. Hell, “4Pi-confocal microscopy of live cells”, Ultramicroscopy. 87, pp. 155-164, 2001

6. Virendra N. Mahajan, Aberration theory made simple, SPIE, 1991

7. Min Gu, Principles of Three-Dimensional Imaging in Confocal Microscopes, World Scientific, 1996 